\title{
Época de semeadura do milho para a região de Sete Lagoas, MG, baseada na probabilidade de ocorrência de períodos secos e chuvosos
}

\author{
Marcos Antonio Vanderlei Silva ${ }^{1}$, Williams Pinto Marques Ferreira ${ }^{2}$, Vanda Maria Sales de Andrade ${ }^{3}$, Samira \\ Gabriela de Almeida Araujo ${ }^{4}$.
}

\section{RESUMO}

O objetivo deste estudo foi determinar as probabilidades de ocorrência de períodos secos e chuvosos na região de Sete Lagoas, MG, a partir de uma série de 66 anos de dados diários de precipitação pluvial, visando subsidiar a definição da melhor data de semeadura do milho. Foram considerados dias secos aqueles que apresentaram precipitação inferior à evapotranspiração do milho, $\mathrm{ET}_{\text {milho }}$ O estudo foi realizado para as fases de floração e enchimento de grãos a partir de sete datas de semeadura DS (01/10, 16/10, 31/10, 15/11, 01/12, 16/12 e 31/12). As chances de ocorrência dos períodos secos e chuvosos foram estimadas mediante o uso da cadeia de Markov. A probabilidade de ocorrência de dias secos foi sempre superior à de dias chuvosos. As maiores possibilidades de ocorrência de dias secos foram observadas entre as DS 15/11 e 31/12. A maior probabilidade de ocorrência de dias chuvosos foi registrada na DS 01/ 10. Considerando o ciclo médio estudado (para a fase mais crítica do milho), a combinação de menor chance de períodos secos com a de dias chuvosos indica que as melhores datas para iniciar a semeadura de sequeiro seriam as de DS 01/10 e 16/10.

Palavras-chave: Milho de sequeiro, cadeia de Markov, déficit hídrico.

\section{ABSTRACT}

\section{Corn sowing time in Sete Lagoas region, Minas Gerais State, based on the probability of occurrence of dry and rainy spells}

The objective of this study was to determine the probability of dry and rainy occurrence periods in Sete Lagoas, Minas Gerais State, Brazil, from a historical series of 66-year-long daily rainfall data to establish the best sowing date for corn crop. Dry days were considered as those that had precipitation below corn evapotranspiration, ET corn $_{\text {. This }}$ study was carried out for the stages of flowering and grain filling, starting from seven sowing times ST (10/01, 10/16, 10/31, 11/15, 12/01, 12/16 e 12/31). The probabilities of occurring dry and rainy spells were estimated by the Markov's chain. The probability of occurring dry days was always higher than rainy days. The highest probability of dry days were found between ST 15/11 and DS 31/12. The highest probability of occurring rainy days was recorded at ST 01/10. Considering the studied average cycle (for the most critical stage of corn development), the combination of the lowest probability of dry spells with the highest probability of rainy days indicates that the best dates to initiate non-irrigated sowing should be ST 01/10 and 16/10.

Key words: Markov's chain, non-irrigated corn, water deficit.

\footnotetext{
Recebido para publicação em maio de 2008 e aprovado em abril de 2010

${ }^{1}$ Engenheiro Agrônomo, Doutor. Universidade do Estado da Bahia, Departamento de Ciências Humanas, Campus 9, BR 242, km 04, Loteamento Flamengo, 47800-000, Barreiras, Bahia, Brasil. maavsilva@uneb.br

${ }^{2}$ Meteorol., Doutor. Empresa Brasileira de Pesquisa Agropecuária, Centro Nacional de Pesquisa de Milho e Sorgo, Milho e Sorgo,Rod. MG 424, KM 45, Caixa-Postal: 285, 35701970, Belo Horizonte, Minas Gerais, Brasil. williams@cnpms.embrapa.br (autor para correspondência)

${ }^{3}$ Meteorol., Mestre. Doutoranda em Metereologia Agrícola na Universidade Federal de Viçosa, Av. P H Rolfs s/n, Campus Universitário, 36570-000, Viçosa, Minas Gerais, Brasil. vanda007@yahoo.com

${ }^{4}$ Estudante de Engenharia Ambiental UNIFFEM, Sete Lagoas, Minas Gerais, Brasil. samiragabiaraujo@yahoo.com
} 


\section{INTRODUÇÃO}

Em Minas Gerais, a cultura do milho tem posição de destaque em razão das diversas aplicações que o cereal tem nas propriedades agrícolas, tanto na alimentação animal quanto na humana e ainda como fonte de renda decorrente da comercialização da produção excedente na propriedade.

Para o ano agrícola 2007/2008, as projeções de produção em Minas Gerais são de aproximadamente 6,3 milhões de toneladas, semelhante ao ano anterior (Rocha, 2008). Segundo Sans \& Santana (2002), o milho, por razões principalmente econômicas, é plantado, na maioria das áreas do Brasil, no período chuvoso, ou seja, é uma cultura típica de sequeiro. A região de Sete Lagoas tem estação chuvosa de outubro a abril, com total médio de 1.234,8 mm, e uma precipitação média anual de 1.328,7 mm (INMET, 2008). Contudo, apesar desse índice pluviométrico anual ser efetivo ao cultivo regular do milho de sequeiro, existe o risco climático a que essa cultura está sujeita devido à irregularidade na distribuição de chuvas, traduzida por veranicos de diferentes durações.

A semeadura de milho envolve riscos de várias ordens, como semente de má qualidade, manejo inadequado de fertilizantes, equipamentos e, principalmente, falta de água. As perdas em produção podem variar com a intensidade e duração do déficit hídrico, sendo mais intensas quando ocorridas durante as fases mais críticas (florescimento e enchimento de grãos), pois, para o milho, as maiores exigências em água concentram-se na fase de emergência, florescimento e formação do grão (Doorenbos \& Kassam, 1994; Fancelli \& Dourado Neto, 2000).

É comum a citação na literatura que as máximas produtividades ocorrem quando o consumo de água durante todo o ciclo varia entre 500 e $800 \mathrm{~mm}$ e, ainda, que a cultura exige de 350 a 500 mm para que ocorra a produção sem necessidade de irrigação. Em condições de clima quente e seco, o consumo de água raramente excede $3 \mathrm{~mm}$.dia ${ }^{-1}$ quando a planta apresenta aproximadamente $30 \mathrm{~cm}$ de altura, e no período que vai da iniciação floral à maturação pode atingir valores de 5 a 7 mm.dia-1 (Sans \& Santana, 2002).

A definição das melhores épocas de semeadura pode ser obtida utilizando técnicas convencionais de experimentação, nas quais se faz a semeadura da cultura em diferentes datas, avaliando-se suas características biométricas e a produtividade alcançada (Marin et al., 2006). No entanto, para a realização de tais pesquisas, além da grande demanda de tempo para sua execução, o custo envolvido é relativamente elevado. Todavia, com o uso da climatologia estocástica, problemas dessa ordem podem ser contornados, já que a previsão de ocorrência de períodos secos é fundamental para planejamento das melhores datas de semeadura, visando à redução do risco para o produtor.

A escolha da época de semeadura é uma forma de selecionar períodos em que as condições climáticas predominantes indicam disponibilidade de água para os períodos críticos da cultura. Alguns estudos já foram conduzidos considerando as probabilidades de ocorrência de períodos secos mediante o uso da cadeia de Markov, admitindo-se a hipótese da persistência em primeira ordem, isto é, que o evento do dia atual depende unicamente daquele do dia anterior. Tal proposição apresentou resultados satisfatórios (Genovez, 1987, Silva et al., 1997; Fietz et al., 1998, Andrade Júnior et al., 2001). Por outro lado, alguns modelos que se baseiam na hipótese de que a precipitação diária é um processo aleatório independente não apresentam bom desempenho (Genovez, 1987). Devido à importância da verificação da ocorrência de déficit hídrico em regiões agrícolas, desenvolveu-se o presente trabalho com o objetivo de determinar as melhores datas de semeadura para o milho, com base na probabilidade de ocorrência de dias secos e chuvosos na fase crítica da cultura para Sete Lagoas, MG.

\section{MATERIAL E MÉTODOS}

A análise estatística foi realizada em uma série de 66 anos (1942 a 2007) de dados diários de precipitação da Estação Climatológica Principal pertencente ao INMET (Instituto Nacional de Meteorologia), localizada em Sete Lagoas, MG (Lat. 19²8', Long. 4500' e Alt. 732 m). Na definição de ocorrências de chuva foram consideradas apenas as precipitações pluviais superiores ou iguais à evapotranspiração diária da cultura do milho, ET $_{\text {milho }}$ (Tabela 1). Assim, um dia foi considerado seco (com déficit de chuva) quando a precipitação foi menor ou igual a $\mathrm{ET}_{\text {milho }}$ e chuvoso na situação contrária. Na análise, considerou-se o número de sequências de dias com e sem chuva dentro da fase FIII, que representa o início do florescimento até a formação de grãos e o final da formação de espigas até o início da maturação, para sete diferentes datas de semeadura DS (01/10, 16/10, 31/10, 15/11, 01/12, 16/12 e 31/12). De acordo com os cultivares de milho mais plantados na região agrícola do município, neste trabalho foi considerado um ciclo de 120 dias (ciclo médio) representando um cultivar hipotético.

Para a estimativa da probabilidade de ocorrência de períodos secos $\mathrm{P}(\mathrm{S})$ e chuvosos $\mathrm{P}(\mathrm{C})$, utilizou-se o processo baseado na cadeia de Markov por meio das seguintes equações:

$$
\begin{aligned}
& P(S)=\frac{\sum D S}{\sum(D S+D C)} \\
& P(C)=1-P(S)
\end{aligned}
$$

em que DS é o dia seco e DC o chuvoso. O cálculo das probabilidades condicionais foi efetuado por meio das equações propostas por Fietz et al. (1998) e Andrade Júnior et al. (2001):

$P(S \mid S)=\frac{\sum D S S}{\sum D S}$

$P(S)=1-P(S \mid S)$ 
Tabela 1. Demanda evapotranspirativa por fase fenológica da cultura do milho

\begin{tabular}{llcc}
\hline & \multicolumn{1}{c}{ Fases dos subperíodos } & $\begin{array}{c}\text { Duração } \\
\text { (dias) }\end{array}$ & $\begin{array}{c}\text { Evapotranspiração } \\
\text { diária (mm) }\end{array}$ \\
\hline FI & Semeadura até a emergência com 20\% de cobertura do solo & 20 & 3,50 \\
FII & Desde 20\% de cobertura do solo até o início do florescimento com IAF máximo & 35 & 4,28 \\
FIII $\quad$ & $\quad$ Início do florescimento até a formação de grãos & 40 & 5,37 \\
& Final da formação de espigas até o início da maturação & 25 & 3,40 \\
\hline
\end{tabular}

Fonte: Albuquerque \& Resende (2002) e Brunini et al. (2006)

$P(C \mid C)=\frac{\sum D C C}{\sum D C}$

$P(S \mid C)=1-P(C \mid C)$

em que DSS é o dia seco precedido de dia seco; DCC o dia chuvoso precedido de dia chuvoso; $\mathrm{P}(\mathrm{S} \mid \mathrm{S})$ a probabilidade de ocorrência de um dia seco, sendo o dia anterior seco; $\mathrm{P}(\mathrm{C} \mid \mathrm{S})$ a possibilidade de ocorrência de um dia chuvoso, sendo o anterior seco; $\mathrm{P}(\mathrm{S} \mid \mathrm{C})$ a probabilidade de ocorrência de um dia seco, sendo o anterior chuvoso; e $\mathrm{P}(\mathrm{C} \mid \mathrm{C})$ a chance de ocorrência de um dia chuvoso, sendo o anterior chuvoso. As probabilidades de ocorrência de dias consecutivos secos, $\mathrm{P}(\mathrm{S}, \mathrm{n})$, ou chuvosos, $\mathrm{P}(\mathrm{C}, \mathrm{n})$, foram calculadas pelas expressões a seguir (Viana et al., 2002):

$P(S, n)=P(S) \times P(S \mid S)^{n-1}$

$P(C, n)=P(C) \times P(C \mid C)^{n-1}$

em que "n" representa o número de dias consecutivos do período considerado.

\section{RESULTADOS E DISCUSSÃO}

As chances de ocorrência de períodos secos apresentados na Tabela 2 permitem identificar duas divisões cronológicas. Entre 01/10 e 31/10, em que a probabilidade P(S) média foi de $66,79 \%$ com uma variação entre as datas de semeadura DS inferior a $1 \%$, e entre $15 / 11$ e 31/12, em que a probabilidade $\mathrm{P}(\mathrm{S})$ foi superior a $70 \%$, com variação entre a DS de aproximadamente $11 \%$. Não obstante esse comportamento, as possibilidades de ocorrerem dias com déficit de chuva foram sempre superiores às de dias chuvosos. A menor chance de dias secos foi verificada na DS 01/10, na qual a probabilidade de ocorrência de dias chuvosos foi aproximadamente a metade da ocorrência de dias secos. Silva et al. (1997) encontraram proporção de 5 para 3 quando estudaram os veranicos para o semestre chuvoso no Cerrado baiano.

Na Figura 1 observou-se que a semeadura em 31/12 apresentou o valor médio do total precipitado na Fase III inferior a $215 \mathrm{~mm}$ (181,87 mm). Espera-se, aproximadamente, que em oito de cada 10 dias $(\mathrm{P}(\mathrm{S})=81,36 \%)$ o milho cultivado possa passar por déficit hídrico no florescimento. A maior probabilidade de ocorrer dias chuvosos foi observada na DS 01/10, com 33,83\%. Registrou-se nessa mesma DS aproximadamente $50 \%$ de chance de ocorrência de dois dias consecutivos de chuva e, consequentemente, possibilidade semelhante de ocorrer um dia seco precedido de um chuvoso (Tabela 2).

A probabilidade de ocorrência de um dia seco sendo o anterior seco $\mathrm{P}(\mathrm{S} \mid \mathrm{S})$ foi aproximadamente $73 \%$ na DS 01/ 10 , aumentando para aproximadamente $86 \%$ na DS 31/12. Nessa época, como se observa na Figura 1, o risco climático aumenta, pois ocorre redução dos totais de precipitação em relação às semeaduras na DS 01/10 com 46\% (157,13 mm), e na DS 16/12 com 19\% (43,14 mm). A chuva na Fase III é fundamental, pois a cultura do milho, em média, apresenta demanda hídrica em torno de $215 \mathrm{~mm}$. De acordo com Bergonci \& Bergamaschi (2002), o maior consumo de água pela cultura de milho é verificado por ocasião do florescimento, já que nessa fase, logo após a emissão da folha-bandeira, o índice de área foliar das plantas é máximo. Assim, a sensibilidade dos processos fisiológicos ligados à formação do zigoto e ao início do enchimento de grãos e, por outro lado, a elevada transpiração que ocorre no mesmo período justificam a consideração do período compreendido entre a emissão da inflorescência masculina e o início da formação dos grãos como sendo extremamente crítico quanto ao suprimento de água (Nied et al.,

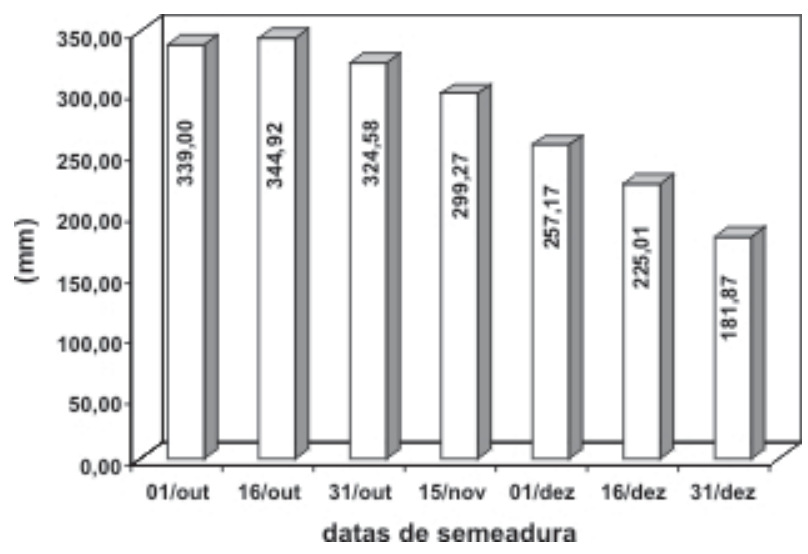

Figura 1. Total médio de chuva (1942-2007) dentro da fase FIII para as respectivas datas de semeadura. 
2005). Dessa forma, ressalta-se que a DS 31/12 não possui quantidade total de precipitação pluvial (média) suficiente para suprir a necessidade hídrica (média) na Fase III desse cultivar hipotética.

Fancelli \& Dourado Neto (2000) relatam que a ocorrência de deficiência hídrica de uma semana durante o florescimento masculino pode implicar em redução de produção próxima de 50\%, enquanto após a polinização nas mesmas condições as perdas podem chegar a $25 \%$. Tratando-se de períodos secos consecutivos, as maiores chances de ocorrência de uma sequência de cinco dias secos foram encontradas entre as DS 01/12 e 31/12, com probabilidades acima de $32 \%$ (Tabela 3). Destaca-se a DS 16/12, com probabilidade $\mathrm{P}(\mathrm{S}, 5)$ de $37,04 \%$, e a DS 31/12, com probabilidade $\mathrm{P}(\mathrm{S}, 5)$ de $45,30 \%$, nas quais espera-se que em um a cada dois anos não ocorram, por cinco dias consecutivos, chuvas acima da $\mathrm{ET}_{\text {milho }}$. Pode-se também prever um déficit de chuva para um período de 10 dias seguidos com probabilidade $\mathrm{P}(\mathrm{S}, 10)$ igual a 15,18 na $\mathrm{DS}$ $16 / 11$ e $\mathrm{P}(\mathrm{S}, 10)$ igual a $21,79 \%$ na DS 31/12. O tempo de retorno de ocorrência desse caso extremo seria de um em cada seis anos e meio e de um em cada quatro anos e meio, respectivamente, para as DS 16/12 e 31/12. Na DS 01/10, considerando essa antecipação de risco de déficit, o tempo de retorno aumentaria para 23 anos.

As chances de chover 10 dias consecutivos foram desprezíveis para as DS estabelecidas neste estudo, apresentando probabilidade $\mathrm{P}(\mathrm{C}, 10)$ inferior a $0,2 \%$

Tabela 2. Data de semeadura (DS), probabilidades de ocorrências de períodos secos (S), períodos chuvosos (C) e probabilidades condicionais na Fase III

\begin{tabular}{lccccccc}
\hline D S & Subperíodo FIII & $\mathbf{P ( S )}$ & $\mathbf{P}(\mathbf{C})$ & $\mathbf{P}(\mathbf{S} / \mathbf{S})$ & $\mathbf{P}(\mathbf{C} / \mathbf{S})$ & $\mathbf{P}(\mathbf{C} / \mathbf{C})$ & $\mathbf{P}(\mathbf{S} / \mathbf{C})$ \\
\hline $01 / 10$ & $25 / 11-03 / 01$ & 66,17 & 33,83 & 73,77 & 26,23 & 48,76 & 51,24 \\
$16 / 10$ & $10 / 12-18 / 01$ & 66,59 & 33,41 & 75,16 & 24,84 & 50,28 & 49,72 \\
$31 / 10$ & $25 / 12-02 / 02$ & 67,61 & 32,39 & 77,62 & 22,38 & 53,02 & 46,98 \\
$15 / 11$ & $09 / 01-17 / 02$ & 70,45 & 29,55 & 79,51 & 20,49 & 51,09 & 48,91 \\
$01 / 12$ & $25 / 01-05 / 03$ & 72,65 & 27,35 & 81,75 & 18,25 & 46,61 & 53,39 \\
$16 / 12$ & $09 / 02-20 / 03$ & 75,61 & 24,39 & 83,66 & 16,34 & 43,29 & 56,71 \\
$31 / 12$ & $24 / 02-04 / 04$ & 81,36 & 18,64 & 86,38 & 13,62 & 40,32 & 59,68 \\
\hline
\end{tabular}

Tabela 3. Probabilidade de ocorrência de períodos secos e chuvosos para 5, 10 e 20 dias consecutivos

\begin{tabular}{lccccccc}
\hline D S & Subperíodo FIII & $\mathbf{P}(\mathbf{S}, \mathbf{5})$ & $\mathbf{P}(\mathbf{S , 1 0 )}$ & $\mathbf{P}(\mathbf{S}, \mathbf{2 0})$ & $\mathbf{P}(\mathbf{C}, \mathbf{5})$ & $\mathbf{P}(\mathbf{C , 1 0 )}$ & $\mathbf{P ( C , 2 0 )}$ \\
\hline $01 / 10$ & $25 / 11-03 / 01$ & 19,60 & 4,28 & 0,0 & 1,91 & 0,05 & 0,0 \\
$16 / 10$ & $10 / 12-18 / 01$ & 21,25 & 5,09 & 0,0 & 2,14 & 0,07 & 0,0 \\
$31 / 10$ & $25 / 12-02 / 02$ & 24,54 & 6,91 & 0,0 & 2,56 & 0,11 & 0,0 \\
$15 / 11$ & $09 / 01-17 / 02$ & 28,15 & 8,94 & 0,0 & 2,01 & 0,07 & 0,0 \\
$1 / 12$ & $25 / 01-05 / 03$ & 32,45 & 11,85 & 0,0 & 1,29 & 0,03 & 0,0 \\
$16 / 12$ & $09 / 02-20 / 03$ & 37,04 & 15,18 & 0,0 & 0,86 & 0,01 & 0,0 \\
$31 / 12$ & $24 / 02-04 / 04$ & 45,30 & 21,79 & 0,0 & 0,49 & 0,01 & 0,0 \\
\hline
\end{tabular}

\section{CONCLUSÕES}

Considerando o cultivar hipotético com ciclo médio de 120 dias para a FIII do milho, as probabilidades de ocorrerem dias com déficit de chuva foram sempre superiores às de dias chuvosos. As maiores chances de ocorrem dias secos foram observadas na data de semeadura DS 15/11 a DS 31/12. A maior probabilidade de ocorrência de dias chuvosos foi registrada na DS 01/10. Para a fase mais crítica do milho, a combinação da menor chance de ocorrer dias secos com a maior probabilidade de dias chuvosos indica que as melhores datas para iniciar a semeadura do milho de sequeiro seriam DS 01/10 e 16/10.

\section{REFERÊNCIAS}

Albuquerque PEP \& Resende M (2002) Cultivo do milho: manejo de irrigação. Sete Lagoas, EMBRAPA-CNPMS,. 8p. (EMBRAPA-CNPMS. Comunicado Técnico, 47).
Andrade Júnior AS, Frizzone JA \& Sentelhas PC (2001) Simulação de precipitação diária para Parnaíba e Teresina, PI, em planilha eletrônica. Revista Brasileira de Engenharia Agrícola e Ambiental, 5:271-278.

Bergonci JI \& Bergamaschi H (2002) Ecofisiologia do milho (compact disc). In: $24^{\circ}$ Congresso Nacional de Milho e Sorgo, Florianópolis. Anais, Florianópolis-ABMS.

Brunini O, Abramides PLG, Brunini APC \& Carvalho JP (2006) Caracterizações macroclimáticas, agrometeorológicas e restrições ambientais para o cultivo de milho em regiões tropicais baixas. InfoBibos, v.1, n.3. Artigo em Hypertexto. Disponível em: http://www.infobibos.com/Artigos/2006_3/ambiente/ index.htm. Acessado em: 10/04/2008.

Doorenbos J \& Kassam AH (1994) Efeito da água no rendimento das culturas. Campina Grande, UFPB. p.3-101. (Estudos FAO: Irrigação e Drenagem, 33).

Fancelli AL \& Dourado Neto D (2000) Produção de milho. Guaíba, Agropecuária. 360p.

Fietz CR, Urchei MA, Frizzone JA \& Folegatti MV (1998) Probabilidade de ocorrência de períodos secos e chuvosos na região de Dourados, MS. Irriga, 3:16-22. 
Genovez AM (1987) Avaliação e regularização de vazões em pequenas bacias com dados esparsos. Tese de Doutorado. EESC/ USP, São Carlos. 168p.

Inmet. Instituto Nacional de Meteorologia (2008) Banco de Dados da Estação Climatológica Principal, Embrapa Milho e Sorgo.

Marin FR, Pandorfi H, Sentelhas PC, Camargo MBP de \& Hernandez FBT (2006) Perda de produtividade potencial da cultura do sorgo no Estado de São Paulo. Bragantia, 65:157-162.

Nied AH, Heldwein AB, Estefanel V, Silva JC da \& Alberto CM (2005) Épocas de semeaduras de milho com menor risco de ocorrência de deficiência hídrica no município de Santa Maria, RS, Brasil. Revista Ciência Rural, 35:995-1002.

Rocha DCC (2008) Milho: bom cenário deve continuar. Agronegócios, Secretaria de Estado de Agricultura, Pecuária e Abastecimento de Minas Gerais. Disponível em: http:/ /www.zootecniabrasil.com.br/sistema/modules/news/ article.php?storyid=1267. Acessado em: 25 de fevereiro de 2008 .
Sans LMA \& Santana DP (2002) Cultivo do milho: clima e solo. Sete Lagoas, EMBRAPA-CNPMS. 4p. (EMBRAPA-CNPMS. Comunicado Técnico, 38)

Silva MAV, Pinto JM, Almeida Júnior JC de, Silva IO \& Soares Neto JP (1997) Estudos dos períodos secos dentro do semestre chuvoso para o cerrado baiano. (compact disc) In: $10^{\circ}$ Congresso Brasileiro de Agrometeorologia, Piracicaba. Anais, Sociedade Brasileira de Agrometeorologia/ESALQ. p.131-133.

Viana TV de, Azevedo BM de, Bomfim GV do \& Andrade Júnior AS de (2002) Probabilidade de ocorrência de períodos secos e chuvosos em Pentecostes, CE. Irriga, 7:226-229. 